\title{
Aldeas y pueblos prehispánicos en la costa de Manabí: Chirije y Japoto
}

Hameaux et villages préhispaniques sur la côte centrale du Manabí: Chirije et Japoto

Prehispanic hamlets and villages on the central coast of Manabi province: Chirije and Japoto

Jean-François Bouchard, Franklin Fuentes y Telmo López

\section{(Q) OpenEdition}

\section{Journals}

Edición electrónica

URL: https://journals.openedition.org/bifea/3615

DOI: $10.4000 /$ bifea.3615

ISSN: 2076-5827

\section{Editor}

Institut Français d'Études Andines

\section{Edición impresa}

Fecha de publicación: 1 diciembre 2006

ISSN: 0303-7495

\section{Referencia electrónica}

Jean-François Bouchard, Franklin Fuentes y Telmo López, «Aldeas y pueblos prehispánicos en la

costa de Manabí: Chirije y Japoto», Bulletin de l'Institut français d'études andines [En línea], 35 (3) | 2006,

Publicado el 29 mayo 2014, consultado el 07 diciembre 2022. URL: http://journals.openedition.org/ bifea/3615 ; DOI: https://doi.org/10.4000/bifea.3615

\section{cc) (1) $\odot$}

Creative Commons - Atribución-NoComercial-SinDerivadas 4.0 Internacional - CC BY-NC-ND 4.0 https://creativecommons.org/licenses/by-nc-nd/4.0/ 
Primera PARTE

Resultados preliminares del Proyecto Manabí Central

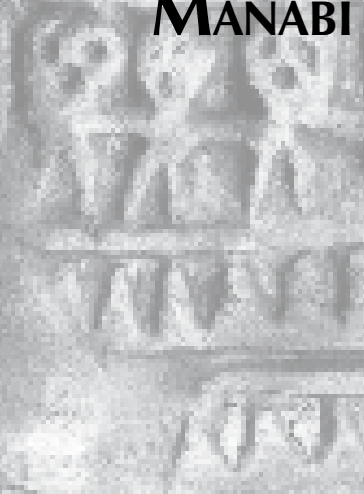





\title{
Aldeas y pueblos prehispánicos en la costa de Manabí: Chirije y Japoto
}

\author{
Jean-François Bouchard* \\ Franklin Fuentes** \\ Telmo López***
}

\section{Resumen}

La ponencia presenta los sitios arqueológicos de Chirije y Japoto, localizados en la costa central de la provincia de Manabí, entre el estuario del río Chone al norte y el estuario del río Porto Viejo al sur. Chirije corresponde a un tipo de asentamiento en la desembocadura de pequeños esteros. Japoto corresponde a un extenso sitio manteño (posiblemente con niveles premanteños), construido en la llanura aluvial del río Porto Viejo. Japoto se caracteriza por ser uno de los pocos sitios en la costa central con grandes y numerosos montículos artificiales (o tolas) preservados. Ambos sitios se estudian dentro de un proyecto arqueológico desde 2003.

Palabras clave: cultura manteña, Ecuador, Manabí, tolas, montículos artificiales

\section{Hameaux et villages préhispaniques sur la côte centrale du Manabí : Chirije et Japoto}

\section{Résumé}

Cet article présente les sites de Chirije et de Japoto localisés sur la côte centrale du Manabí, entre I’estuaire du río Chone et celui du río Porto Viejo. Le premier correspond à un modèle d'établissement au débouché de cours d'eau saisonniers. Le second correspond à un grand site manteño et peut être

* CNRS. Nanterre-Cedex (París, Francia). E-mail: jean-francois.bouchard@mae.u-paris10.fr

** Arqueólogo. Contraparte nombrada por el Instituto Nacional de Patrimonio cultural (INPC) del Ecuador para el sitio Chirije. E-mail: ffuentes@yahoo.com

${ }^{* * *}$ Arqueólogo. Contraparte nombrada por el Instituto Nacional de Patrimonio cultural (INPC) del Ecuador para el sitio Japoto. E-mail: tefelomu@yahoo.com 
pre-manteño, près de l'embouchure du río Porto Viejo et constitue l'un des rares exemples encore préservés d'établissement sur monticules artificiels sur le littoral équatorien. Les deux sites font I'objet d'un projet archéologique depuis 2003.

Mots clés : culture manteña, Équateur, Manabí, tolas, monticules artificiels

\title{
Prehispanic hamlets and villages on the central coast of Manabi province: Chirije and Japoto
}

\begin{abstract}
This paper deals with prehispanic sites, Chirije and Japoto, both located on the seashore or nearby the shore of the Pacific ocean, between the mouth of the Chone river at north, and the mouth of the Porto viejo river at South. Chirije appears to be a small hamlet, tipical of the cliffy coast, located at the very mouth of an «estero» ( seasonal stream or wadi). Japoto, on the contrary, appears to be a large village, with many articial mounds (locally called «tolas»). It stands on the right side of the lowlands at the mouth of the river. It is one of the best sites of the manteña culture that remains well preserved by the present time. Both sites are studied by an archaeological project since 2003.
\end{abstract}

Key words: manteña culture, Ecuador, Manabi, artificial earth mounds, «tolas»

\section{INTRODUCCIÓN}

El proyecto Manabí se dedica al estudio arqueológico de un sector central de la costa de esta provincia de Ecuador, aproximadamente ubicado entre el río Chone (al norte) y el río Porto Viejo (al sur). Estos son los mayores ríos de la región, mientras otros denominados «esteros» son arroyos que solamente tienen agua durante las épocas de invierno. La gran mayoría de la costa está conformada por acantilados altos (más de 30 metros de altura) que llegan hasta el mar en marea alta. Solamente existe una zona más baja a nivel de las desembocaduras de los esteros 1 . Sin embargo en la parte sur, a nivel del valle del río Porto Viejo, existe una llanura más extensa que se extiende desde San Clemente al Norte y Crucitas al sur, en la margen izquierda del río. En esta parte, se presenta un cordón litoral arenoso, playas bajas y un valle bajo que se beneficia del río y de sus aluviones (figs. 1-2).

Por lo tanto podemos definir 2 clases de medio natural: al norte, la costa de acantilados y, al sur, la costa baja y arenosa, sin acantilados, atravesada por el río.

1 Entre Bahía de Caráquez y San Clemente, hay varios esteros que reciben distintos nombres según la gente del lugar o según los mapas del I.G.M. (1/50 000). La información local indica de norte a sur: estero La Bellaca, también llamado La Gringa, (que comunica a Bahía por una carretera asfaltada desde la playa La Bellaca), al norte de la Punta La Gorda, los esteros Chirije grande y Chirije chico, el Norte y El Bálsamo (conectado por un camino a la vía asfaltada hasta el Niño del 1997-1998), ambos al norte de la Punta Viquin. Luego existe un pequeño estero llamado Norte, antes de llegar al pueblo de San Clemente.

En los 3 mapas del IGM (MIII-D3, MIII-F1, MIII-E2, 1/50 000), de norte a sur, aparecen mencionados los esteros El Bejuco, Los Navíos (al norte de Punta La Gorda), luego los esteros La Gorda, El Pajonal, Las Quebraditas, Chirijo, El Bálsamo y un estero sin nombre cerca de San Clemente. Es de notar que frente a Chirije se menciona una Punta Verde, que no se detecta en el paisaje sino por un conjunto de pequeñas rocas esparcidas en la playa. Estos mapas indican también el nombre de Punta de Charapoto para la punta que se nos designó también como Punta Viquin un poco al norte de San Clemente. Al observar los mapas, se nota que solamente los esteros Pajonal y Bálsamo parecen sobrepasar los 5 kilómetros de largo. 
Aldeas y pueblos prehispánicos en la costa de Manabí: Chirije y Japoto

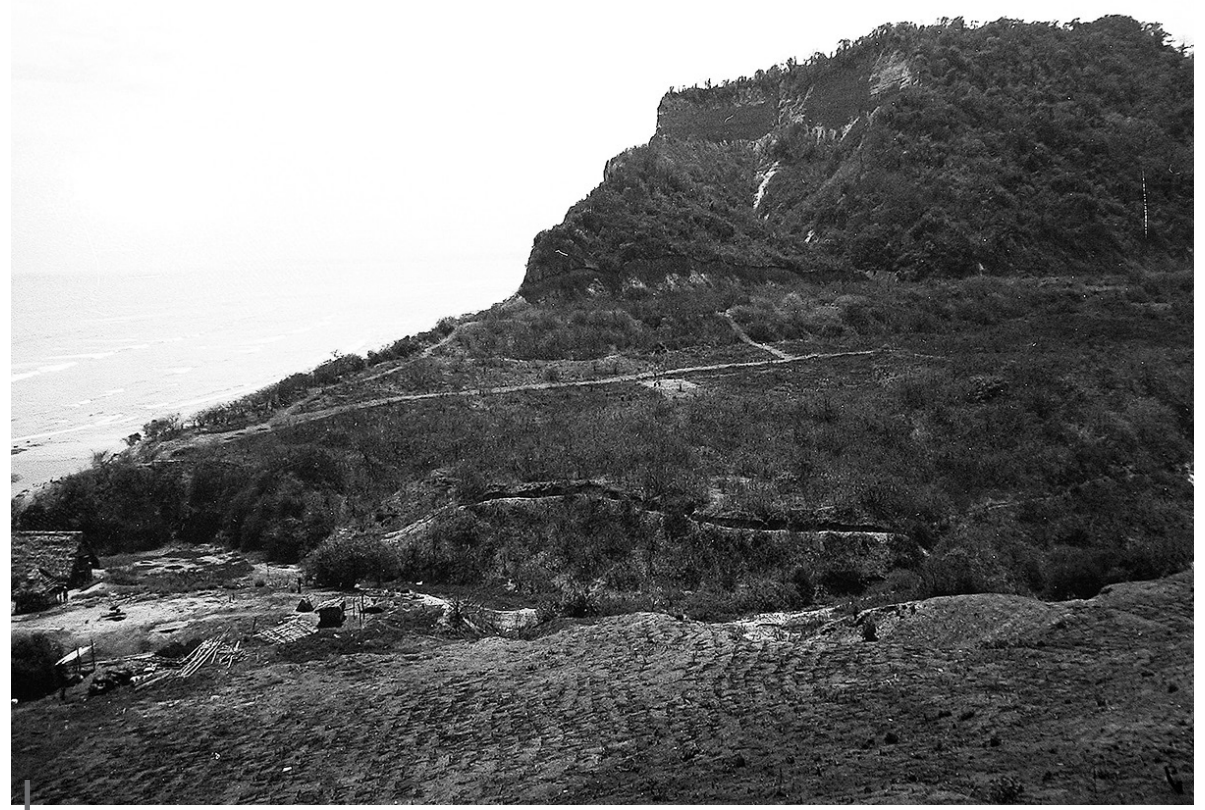

Figura 1 - Chirije, vista hacia el norte

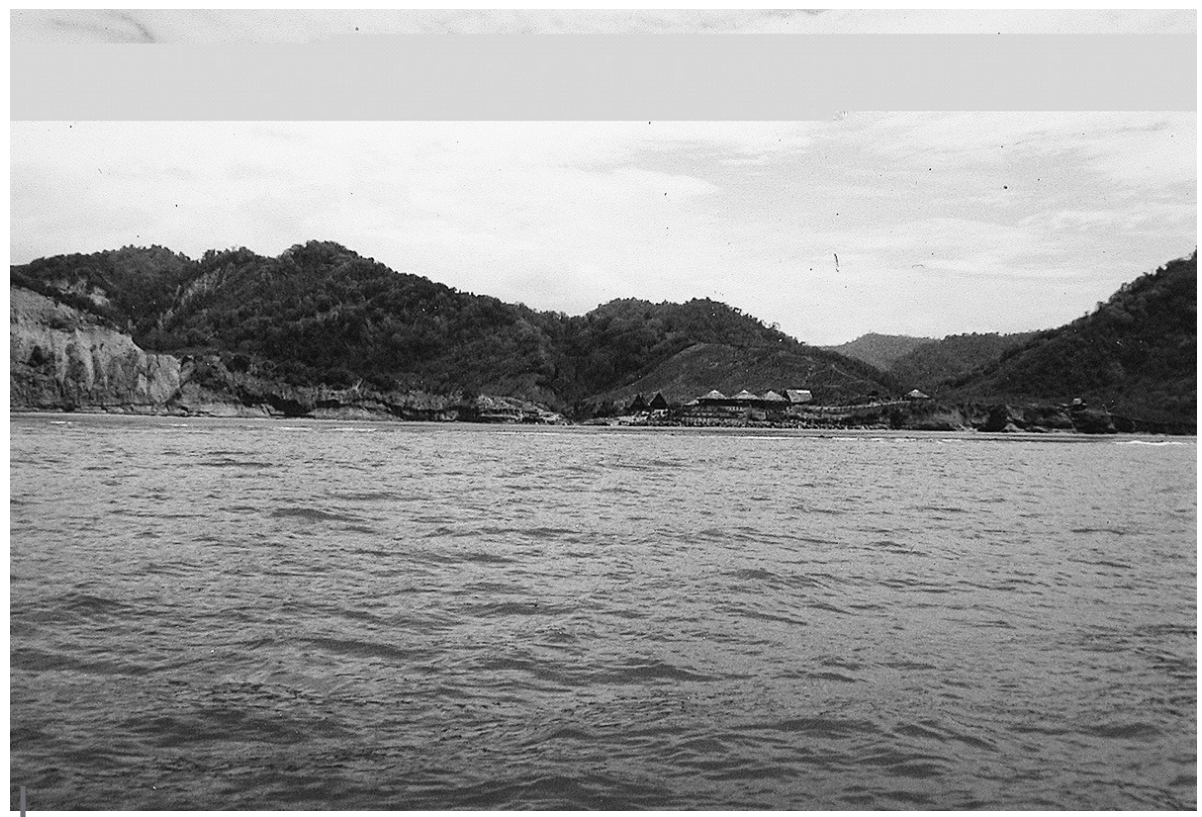

Figura 2 - Chirije, vista desde el mar 
Son pocos los estudios arqueológicos en esta área: mencionaremos como mayores referencias las publicaciones de Marshall Saville $(1907 ; 1910)$ y de Emilio Estrada (1957; 1962), aunque ambos autores se han preocupado más por los sitios de la costa sur de Manabí. Sin embargo, en relación con otras regiones de la costa ecuatoriana, se trata de una zona poco conocida, a parte de los numerosos hallazgos (sobre todo de cerámica) hechos por los huaqueros sobre todo. Tal vez esto se debe a la ausencia (hasta la fecha) de sitios precolombinos «ricos», que son los que atraen a los buscadores de «tesoros» o de vestigios de valor que se puedan vender a coleccionistas. Llama la atención que para nuestra área de estudio, casi no se conocen objetos «de valor» en las colecciones, mientras un sitio como San Isidro (al norte de Bahía de Caráquez), fue intensamente huaqueado y saqueado.

Realmente, era casi una tierra de nadie entre las costas del territorio Jama Coaque y del territorio Manteño Huancavilca. Nuestro objetivo fue intentar poner en relieve las evidencias de ocupaciones prehispánicas que pudieron existir antes del siglo XVI, sea en base a vestigios construidos (las tolas) o artefactos manufacturados.

\section{ASENTAMIENTOS EN LA DESEMBOCADURA DE LOS ESTEROS: EL CASO DE CHIRIJE}

\section{1. Secuencia cultural de Chirije según las evidencias arqueológicas anteriores 2}

\section{1. 1. Los niveles superiores (segunda parte del Periodo de Integración)}

Estos niveles superiores obtenidos en las dos extremidades $\mathrm{N}$ y $\mathrm{S}$ del sitio parecen corresponder a la época de los «corrales» descubiertos por Estrada. Estos corrales manteños son construcciones de planta cuadrangular de unos 15 metros de lado, que se construyeron con piedras cilíndricas perforadas en su centro en toda su altura ${ }^{3}$ que él llama «columnas talladas». Eran enterradas en el piso y solamente se veían sus partes superiores. Se supone que en los huecos se clavaban pequeñas vigas que formaban un armazón para paredes de bahareque. Según Estrada, los corrales serían los vestigios de un centro ceremonial secundario de los «manteños del norte», construido sobre una anterior ocupación. Él encontró un piso preparado manteño (de tierra rojiza con paja) y entierros directos del periodo manteño bajo este piso superior.

En la actualidad, en Chirije, no quedan corrales con huellas visibles in situ ni se puede localizar con precisión su ubicación en relación con el mapa de la publicación de Estrada (1962: 134, fig. 28).

2 Básicamente, para esta secuencia, nos referimos a los estudios de Estrada (1962).

3 Muchas piedras perforadas están agrupadas a la entrada del museo de sitio, junto con un ejemplo moderno de la pared de bahareque con estacas en las piedras para restituir la forma de construcción de los edificios llamados corrales. Otras delimitan ahora pasillos modernos. Es de aclarar que no son «artefactos»: son piedras naturales que presentan una perforación cilíndrica hecha por grandes gusanos marinos en épocas geológicas. Fueron solamente aprovechadas tal cual en la época prehispánica como adecuados elementos de construcción sin modificarlos. En cuanto al uso ceremonial de los corrales, no hemos encontrado más elementos diagnósticos. Por lo tanto, no podemos discutir la propuesta hecha por Estrada quien hipotetiza dicho uso ceremonial. El camino de piedra para llegar a uno de los pozos, mencionado por Estrada, tampoco parece ser un argumento decisivo para poder sugerir que dicho pozo pudo ser la piscina sagrada de los manteños de la época de oro de Chirije (Estrada, 1962: 26). 


\section{1. 2. Los niveles intermedios (primera parte del Periodo de Integración)}

Con este término «niveles intermedios» designamos los niveles que se han descubierto debajo del nivel supuestamente manteño y que yacen desde una profundidad variable según los lugares excavados. Las palabras mismas de Estrada dicen que yacen debajo del «piso manteño», y se trata de «restos habitacionales con piso preparado con barro y paja uniendo curiosas ollas o tinas de barro cocido» (Estrada, 1962: 26). Según el autor, se trata de «un complejo cerámico y cultural diferente del conocido manteño, mereciendo por lo tanto su nombre propio» (Estrada, 1962: 76). Corresponde a la ocupación por la gente que habitaba el sitio durante su periodo inicial. Estrada atribuye estos vestigios a una tradición Chirije, cuya cerámica se encontró también en algunos sitios de Manabí central: el Bálsamo, Cerro Jaboncillo, La Sequita (Pepa de Huso), y, más lejos, en Chanduy (con una fecha ${ }^{14} \mathrm{C}$ de 1200 AD), Playas y San Mateo (Estrada, 1962: 77). Por lo tanto, se le podría asignar una ubicación cronológica dentro del Periodo de Integración, aunque no sea muy precisa. Desde esta época (1962), no se ha podido caracterizar más esta tradición Chirije.

Al mismo tiempo, Estrada atribuye la presencia de grupos caracterizados por la cerámica Chirije a un periodo cronológico que se llama ahora «Periodo de Integración», indicando una posible fecha final alrededor de 1100 AD para estos grupos con céramica Chirije. Pero también relaciona la cerámica Chirije con las dos fases del periodo de Desarrollo Regional (Guangala y Jama Coaque), que supuestamente terminan alrededor de $500 \mathrm{AD}$. Curiosamente, no la relaciona con la fase llamada Bahía de Caráquez.

Todo al contrario, Estrada supone que hubo un largo vacío cultural entre Bahía de Caráquez y Chirije, y al mismo tiempo atribuye el abandono de la región de Bahía de Caráquez a un importante fenómeno volcánico que ocurrió al final del periodo de los Desarrollos Regionales y habría tenido efectos gravísimos sobre las poblaciones de esa cultura Bahía.

«Tal vez los movimientos tectónicos y volcánicos al final de Bahía impidieron vida alguna en esa zona por algunos sitios. Tal vez fue necesario ese largo periodo para lavar suficientemente las tierras y hacerlas nuevamente útiles para la agricultura y la vida del hombre». (Estrada, 1962: 78)

Esto debería contrastarse con la interpretación más reciente dada por J. Zeidler en cuanto a los efectos de las erupciones volcánicas de la región vecina de San Isidro sobre la ocupación Jama Coaque (Zeidler \& Pearsall, 1994: 200-215). En varias oportunidades Estrada emite dudas sobre la procedencia de los grupos que se caracterizan por la cerámica Chirije:

«No sabemos aún positivamente de donde llegaron o se desarrollaron los pueblos del periodo Chirije (...) Es muy posible que en el sector Guangala haya evolucionado esta cultura con la ayuda de elementos extranjeros». (Estrada, 1962: 77)

«En Manabí central, o sea en territorio de la cultura Bahía, Chirije aparece mezclado con elementos como figurillas de la cultura Guangala (...) y cerámica de Jama Coaque, lo que hace suponer un contacto con las fases últimas de Guangala y de Jama Coaque». (Estrada, 1962: 78)

\section{1. 3. Los niveles inferiores (Periodo de Desarrollo Regional)}

Para el sitio de Chirije, es de notar que Estrada no señala una ocupación Bahía y solamente reconoció dos ocupaciones sucesivas: Chirije y Manteña. Por otro lado, aunque no tenemos conocimiento de las conclusiones sintéticas que se han sacado de excavaciones recientes en Chirije (1995), parece que en un pozo de la unidad A3, se han determinado tres momentos de ocupación relacionados con la cultura Bahía de Caráquez (tardío, intermedio y antiguo)4. Esto indicaría una tercera ocupación más antigua que las dos descubiertas inicialmente por Estrada.

4 En 1995, un grupo de estudiantes en arqueología de Guayaquil «Arku», intentó realizar un estudio del sitio Chirije. Se desconocen sus interpretaciones y conclusiones, que hasta la fecha no se han publicado. 
De ser así, sumando los dos resultados, tendríamos hipotéticamente tres grandes momentos de ocupación sucesivos en el sitio Chirije. En orden cronólogico: Bahía-Chirije-Manteño.

Sin embargo, hemos descubierto en nuestras excavaciones algunos indicios que apuntan a otras posibilidades, aún por determinar con mayor precisión:

- En el corte del estero se descubrió un sello cilíndrico que podemos equiparar a unos casi iguales publicados en el estudio dedicado a sellos de la cultura Jama Coaque (Cummins et al., 1996: 139-150).

- También, en el material obtenido en los dos sondeos realizados a unos metros de esta excavación, aparecieron dos otros sellos: uno cilíndrico y un estampador rectangular, que parecen de diseño Jama Coaque (ver Cummins et al., 1996: 196-197).

- Igualmente, un figurín antropomorfo sin cabeza fue descubierto por nosotros en los depósitos de «basureros» de este mismo corte. Este figurín se parece morfológicamente más a los de las áreas costeras norteñas de la costa ecuatoriana (por ejemplo: Jama Coaque, La Tolita) que a los de la costa central del Ecuador (Bahía, Guangala).

La presencia de estos vestigios en contextos arqueológicos bien puede indicar que la ocupación del periodo de Desarrollo Regional en Chirije ha recibido unos aportes foráneos a través de contactos con otras culturas de la costa ecuatoriana, probablemente por vía marítima. Así se podría confirmar que el estuario del río Chone no fue un límite tan estricto como se pensaba entre las culturas Jama Coaque y Bahía de Caráquez. O sea que la ocupación más antigua de Chirije indicaría más bien que, por lo menos a nivel de los objetos de cerámica (a parte de la vajilla) la influencia norteña desde la región Jama Coaque puede parecer predominante (figs. 3-4).

Desde luego, en base a estos limitados indicios, no se puede afirmar que esta ausente la evidencia de una cultura Bahía de Caráquez en Chirije, pero parece obvio que no hay fronteras bien delimitadas ni diferencias abruptas a través de los vestigios materiales y qué vestigios de estilo norteño se encuentran en este sitios.

\section{2. Los asentamientos de playa: propuesta de interpretación}

El aspecto actual nos puede aportar algunos indicios para interpretar los asentamientos prehispánicos a la orilla de playas. Entre otros aspectos es importante observar que el efecto de los fenómenos del Niño en este sector de la costa, si bien causan catástrofes en muchas partes, por las inundaciones y los deslizamientos bien conocidos, también tienen efectos y consecuencias más positivos sobre la vegetación de las áreas más áridas. Gracias a la fuerte humedad generada por los eventos Niño, éstas áreas llegan a cubrirse de vegetación que puede perdurar algún tiempo durante los ciclos inter-Niño. Inclusive, en casos de Niños fuertes, como ocurrió en 1997-1998, se genera probablemente un fuerte aumento de vegetación, llegando a formarse pequeños bosques de árboles y arbustos capaces de resistir luego la sequía durante varios años. Si la frecuencia de Niños sigue un ciclo relativamente estable y no se dan fuertes y prolongadas sequías durante los periodos inter-Niño, es probable que esta franja costera presente condiciones favorables de vegetación para permitir la presencia de asentamientos humanos de tipo tradicional y rústico, como podían ser las aldeas de agricultores prehispánicos. Además, durante los años normales (sin Niño), el volumen anual de precipitaciones de la zona de costa cerca de Bahía de Caráquez (de unos $600 \mathrm{~mm}$ ) es suficiente, a pesar del déficit hídrico importante (alrededor de $800 \mathrm{~mm}$ ), para desarrollar actividades de cultivo de maíz, de yuca o de maní, como se puede apreciar en algunas chacras locales. Podemos considerar que se trata de un medio naturalmente apto para el cultivo de plantas alimenticias, aunque en la actualidad se lo está explotando seguramente muy por debajo de su potencial de producción. La producción prehispánica pudo permitir sustentar una aldea a orillas del mar con acceso —además- a buenas fuentes de proteínas marinas (pescado, crustáceos, mariscos). 
Aldeas y pueblos prehispánicos en la costa de Manabí: Chirije y Japoto

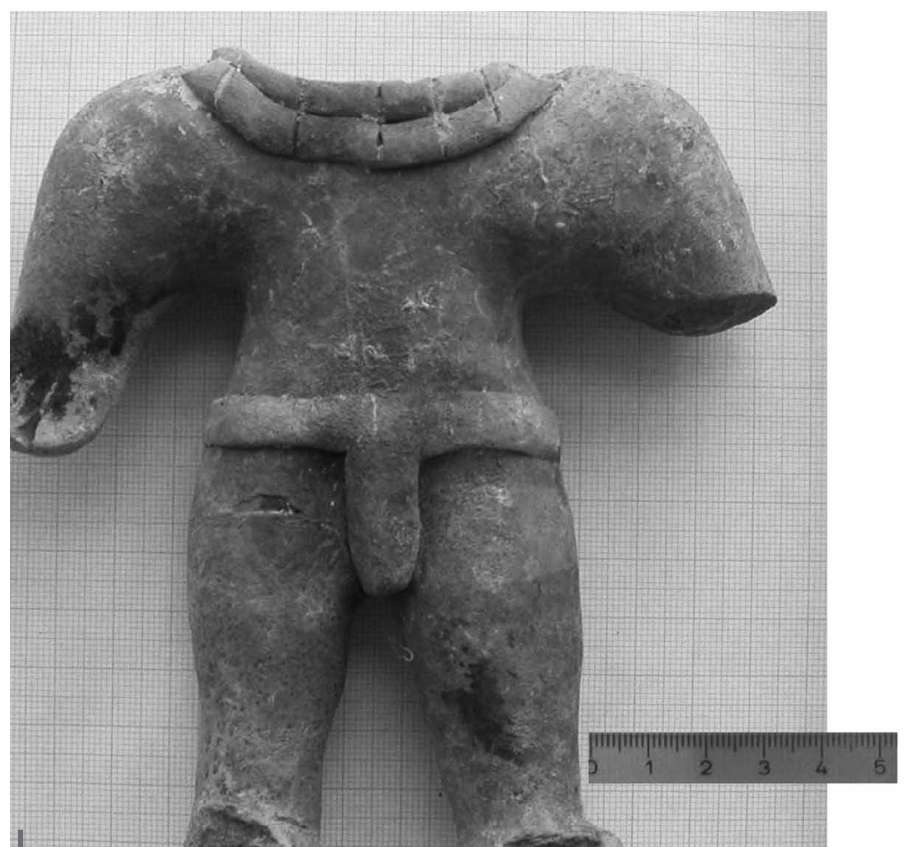

Figura 3 - Chirije: cuerpo de figurín antropomorfo (Desarrollo Regional)

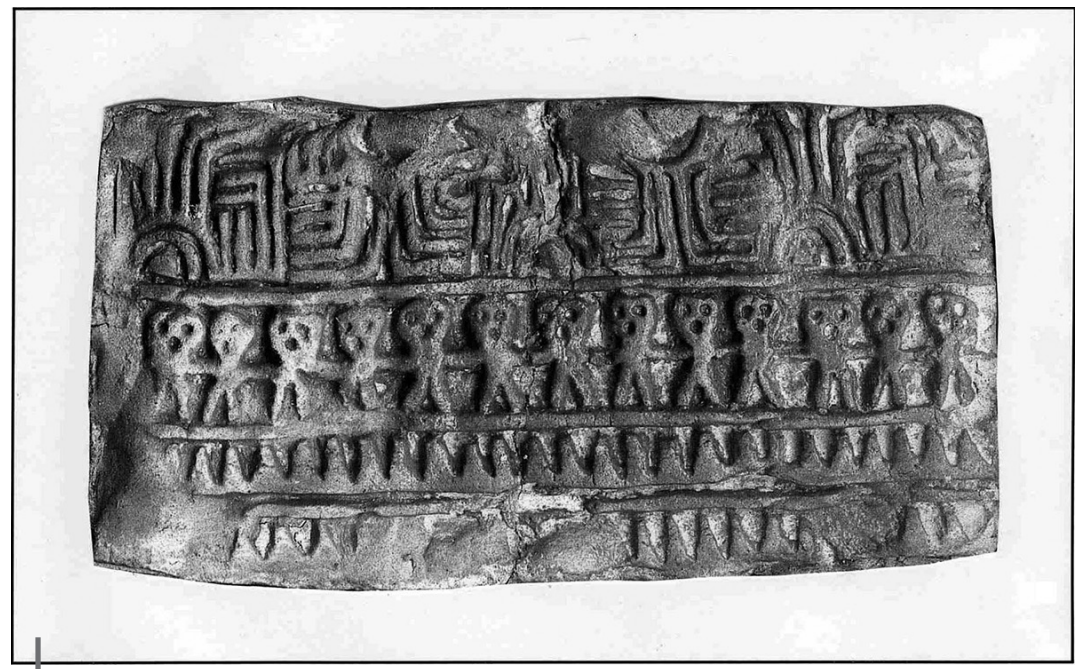

Figura 4 - Chirije: impronta de un sello cilindrico (Desarrollo Regional) 
Nos parece muy probable que el casi abandono de la zona en nuestros días se pueda explicar por factores culturales más que naturales: ausencia de vías, falta de agua dulce y de energía eléctrica, así como la propiedad privada de los terrenos más aptos para asentamientos en las desembocaduras de los esteros.

En los tiempos prehispánicos, tanto en Chirije como en los otros sitios arqueológicos que se han señalado a lo largo de esta costa entre Bahía de Caráquez y San Clemente, el clímax de vegetación consecutiva a un evento Niño, combinado con los ciclos climáticos «normales» inter-Niño, habría permitido el desarrollo de una pequeña red de aldeas costeras ubicadas en cada desembocadura de los esteros.

En resumen: a pesar de la escasa irrigación por los ríos, en condiciones climáticas normales, las precipitaciones anuales en este área parecen siempre suficientes para obtener los principales alimentos vegetales de consumo habitual: maíz, yuca, maní, etc. Estas poblaciones prehispánicas aprovecharon muy bien la vegetación cíclica natural, demostrando así su capacidad de adaptación para asentarse en esta parte costera. Al mismo tiempo, el mar permitía obtener grandes cantidades de proteínas (pescado, crustáceos, mariscos), tal como lo podemos observar en los depósitos a través de abundantes restos de conchas, caracoles marinos y huesos de pescado. Por otra parte, es preciso subrayar que la gran cantidad de materias orgánicas aportadas por el estuario del Chone, a unos 15 kilómetros al norte y por el río Portoviejo, a unos 15 km al sur, enriquece las aguas marinas del área costera y favorece la presencia de una abundante fauna marina en toda esta faja de costa. Podemos hacer comparaciones con la situación actual: se observa en la mayoría del tiempo una fuerte presencia de pequeñas embarcaciones de pesca artesanal que provienen sea del sur (San Clemente) o sea del norte (Bahía de Caráquez) frente a la playa de Chirije (y otras del área). Esto ocurre casi todos los días cuando el estado del mar lo permite. Además, observamos la presencia de varios pescadores de crustáceos, pulpos, mariscos y caracoles marinos en las rocas de las playas, lo que ofrece un aporte global de proteínas marinas nada despreciable.

En la actualidad, sin embargo, la ausencia de viviendas permanentes de pescadores en toda la costa entre Bahía de Caráquez y San Clemente nos oculta las potencialidades del área, contrastada con la actividad económica al sur y al norte. Pero, sugerimos que la forma prehispánica de aprovechar los recursos fue distinta por varias razones.

Primero, en las diversas épocas de ocupación indígena prehispánica era prioritaria la subsistencia de los moradores más que el abastecimiento de otras áreas. No hay duda que, antes de todo, los cultivos y los productos de la pesca servían primero para alimentar a los moradores de los asentamientos costeros. Es decir que se buscaba una auto alimentación de los pobladores para establecer una aldea. Sin embargo, podían «exportar» un eventual excedente hacia el interior, sea en forma fresca, sea en forma elaborada para su conservación.

En este último caso, la presencia de numerosos fogones descubiertos en las excavaciones (tanto en las nuestras como en las anteriores) podría ser un indicio de técnicas de conservación mediante secado y ahumado de los pescados, aunque no hay claros indicios de grasa animal en ellos.

Estrada enfatiza el uso de «tinas de barro cocido mezclado con paja» (Estrada, 1962: 77, fig. 33). Según toda probabilidad, pensamos que se trata de las estructuras que aparecieron en nuestra excavación de la parte central de una trinchera larga. Se trata de huellas de tierra rojiza quemada, con paredes altas y verticales. Si se los compara con la figura 33 que presenta Estrada para el corral B, corte 2, se observa una misma concentración de estructuras de tierra cocida, agrupadas como si se tratará en ambos casos de áreas especializadas.

Estos pueden ser los indicios más evidentes de técnicas de preparación (y/o de conservación) de alimentos marinos o bien de costumbres de quemar alguna materia combustible con frecuencia y en gran cantidad con fines desconocidos hasta ahora 5 .

5 También, observamos estructuras parecidas o semejantes en una excavación realizada en 1995 por el grupo Arku, en la margen derecha del estero Chirije grande, la cual se dejó al aire libre. 
Por otra parte, es interesante notar que no se reportan elementos de molienda (manos de moler y metates) y que tampoco estos han aparecido en nuestras excavaciones. Al mencionar manos de moler para Chirije, Estrada apunta a una herramienta específica hecha de «barro cocido» para obtener pasta de alimento como el ají (Estrada, 1962: 76) y en ningún caso habla de manos de piedra, como las que suelen usarse para moler cereales (entre otros, maíz), aunque si figuran 2 metates en la figura 33 de su libro.

Desde luego, no inferimos del todo que las ocupaciones costeras prehispánicas en este sector se han desarrollado en «autarquía» sin contactos con otras regiones. Al contrario, la presencia de cerámica posiblemente ajena apunta a dichos contactos. Igualmente, podemos suponer como una hipótesis lógica que la gran cantidad de pescados y otros alimentos marinos disponibles sobrepasaba las necesidades del consumo local. Estos excedentes de pesca podían mandarse hacia el interior, como parte de un sistema sencillo de intercambios complementarios de bienes de consumo alimenticio o como parte de un sistema económico aún más complejo.

Estas conclusiones preliminares permiten suponer una probable integración de las poblaciones estrictamente costeras a un sistema más global del Manabí central, en el cual los grupos de la franja marítima formaban parte de un sistema elaborado de explotación de los distintos nichos de la «macrorregión».

\section{JAPOTO: UN PUEBLO DE LA LLANURA EN LA CUENCA BAJA DEL PORTO VIEJO}

Es preciso recordar otra vez que la arqueología de esta parte central de la costa de Manabí no se ha estudiado tanto como otras regiones costeras del Norte o del Sur. Los cronistas del siglo XVI mencionan que al llegar a estas costas al principio de la conquista, los primeros españoles descubrieron un región costera rica, densamente habitada por grupos autóctonos y autónomos que no formaban parte integral del imperio inca. Frecuentemente, los españoles encontraron numerosas poblaciones indígenas, pertenecientes al grupo llamado «manteño», que controlaba gran parte de la costa ecuatorial y del comercio marítimo a media y larga distancia desde grandes asentamientos como por ejemplo Salangone (costa sur de Manabí).

En la literatura, se ha hablado muchas veces de una «liga de mercaderes marítimos» precolombina que practicaban el tráfico de la concha del Spondylus, de metales (cobre, oro) de textiles y materias primas. Según algunos autores, sus embarcaciones, hechas de troncos de balsa y equipadas de velas, alcanzaban las costas de Meso-América y de Perú.

En el siglo XX, varios sitios manteños importantes, especialmente sitios ceremoniales, se han descubierto y estudiado. Muchos objetos y obras de arte destacadas provienen de estos lugares manteños. Sin embargo, no se conocen mayores detalles sobre sitios habitacionales: se menciona Jocay (la actual ciudad de Manta) como un gran asentamiento, lastimosamente muy poco estudiado y probablemente destruido o recubierto por las casas modernas.

Por lo tanto, nuestro actual proyecto se ha dedicado a estudiar un sitio llamado «Japoto»6 que presenta una gran cantidad de montículos artificiales y material manteño en la superficie. El INPC (sede Guayaquil) autorizó este estudio desde finales de 2003. En 2004, 2005 y 2006, 3 temporadas de excavación se han llevado a cabo en los meses de junio-julio.

El sitio arqueológico se ubica a unos $2 \mathrm{~km}$ del pueblo costero San Jacinto (GPS 0¹7'37" sur; $80^{\circ} 30^{\prime} 36^{\prime}$ oeste). Se encuentra muy cerca de la desembocadura del río Porto Viejo, en su margen derecha. La misma presencia del río forma en esta costa una parte baja ancha, que contrasta con las colinas y los acantilados que caracterizan el resto de la costa entre los modernos

6 Para su nomenclatura oficial, el INPC ha decidido retomar el nombre antiguo de «Japoto» para este sitio, antes llamado «Lomas de Charapoto», «Lomas de Santa Teresa», o sencillamente «San Jacinto». Este topónimo Japoto sería el nombre vernacular del sitio, corrompido desde la conquista en «Charapoto». 
puertos de Bahía de Caráquez y Manta. Hemos podido comprobar la existencia de varios vestigios arqueológicos sobre una amplia superficie que alcanza tal vez unas 60-80 hectáreas. Nuestras excavaciones se localizan en una finca de propiedad privada de 19,7 hectáreas; al sur, al norte y al este se aprecian varias tolas7. En nuestra época moderna, al oeste, la creación de piscinas de acuicultura ha destruido los vestigios y al norte la creación muy reciente de extensas salineras acaba de destruir una enorme superficie de terrenos — probablemente en su mayoría- arqueológicos. Aunque el sitio se puede calificar como bien conservado pudimos ver que fue intensivamente explorado por huaqueros (en todas las tolas se presentan varios huecos exploratorios). En la misma finca, los trabajos agrícolas no aseguran una buena conservación de los vestigios, puesto que varios montículos también se han arado y cultivado, a menudo con tractor y maquinaria pesada (figs. 5-6).

Se estima más de 60 montículos artificiales (o tolas), las estructuras manteñas visibles en todo el sitio y sus alrededores8. Estas tolas son de varias formas y varias dimensiones. Hay tolas muy grandes, de plano rectangular, con una plataforma superior claramente horizontal, que pueden medir hasta 4 metros de altura, $60 \mathrm{~m}$ de largo y $20 \mathrm{~m}$ de ancho. Hay tolas más pequeñas, a veces tan altas (o más), con planta circular u ovalada. Hasta ahora no reconocemos una orientación preferencial pero si observamos que muchas fueron construidas próximas a pequeños esteros que contienen agua durante la corta temporada de lluvias (ver la ponencia de P. Usselmann).

\section{1. Principales temas estudiados}

\section{1. 1. Estudio de la cronología cultural}

Los vestigios visibles pertenecen a la cultura manteña, última fase cultural prehispánica del área si consideramos que la mayor parte de la costa fue prácticamente libre de la dominación inca ${ }^{9}$. Es muy probable que la secuencia de ocupación corresponda a por lo menos dos épocas manteñas (Antigua y Tardía). El estado actual de los montículos sería desde luego el reflejo de la última ocupación. Dos dataciones radio carbónicas de la temporada 2004 han dado fechas entre 1000 AD y 1200 AD para niveles intermedios.

\section{1. 2. Modelos de asentamiento y estructura de las tolas}

Nos parece que hubo por lo menos dos momentos sucesivos: al principio, los montículos no eran tan altos y fueron elevados con mucho cuidado en varias etapas, alternando pisos de relleno y suelos habitados. Luego, parece que la última etapa consistió en un relleno masivo, menos cuidadoso, amontonando una sola capa gruesa de sedimento.

Este asentamiento de gran tamaño fue precisamente localizado en un sitio que daba fácil acceso tanto al medio terrestre como al medio acuático. Su importancia puede corresponder a la sede de un poder regional en la rica llanura de Charapoto, para controlar a la misma vez excelentes tierras agrícolas con buena posibilidad de riego y los recursos acuáticos del mar y

7 Sin entrar en discusiones de detalles, designamos como tola un montículo artificial con evidencias de ocupación prehispánica (u otro uso antrópico).

8 A lo largo de un camino secundario que va desde la finca a la carretera San Clemente-Bahía, hemos podido ver también numerosas tolas que pueden formar parte del sitio propriamente dicho o ser parte de un sitio muy cercano y satélite. Igualmente, hemos visto en toda la llanura entre la finca y la moderna carretera que pasa por San Jacinto y San Clemente, numerosos vestigios arqueológicos. Sin embargo la construcción de varias salineras industriales de gran magnitud ha destruido muchos vestigios en los últimos años.

9 No hemos encontrado hasta la fecha nigún vestigio inca ni colonial en el área estudiada. 
Aldeas y pueblos prehispánicos en la costa de Manabí: Chirije y Japoto
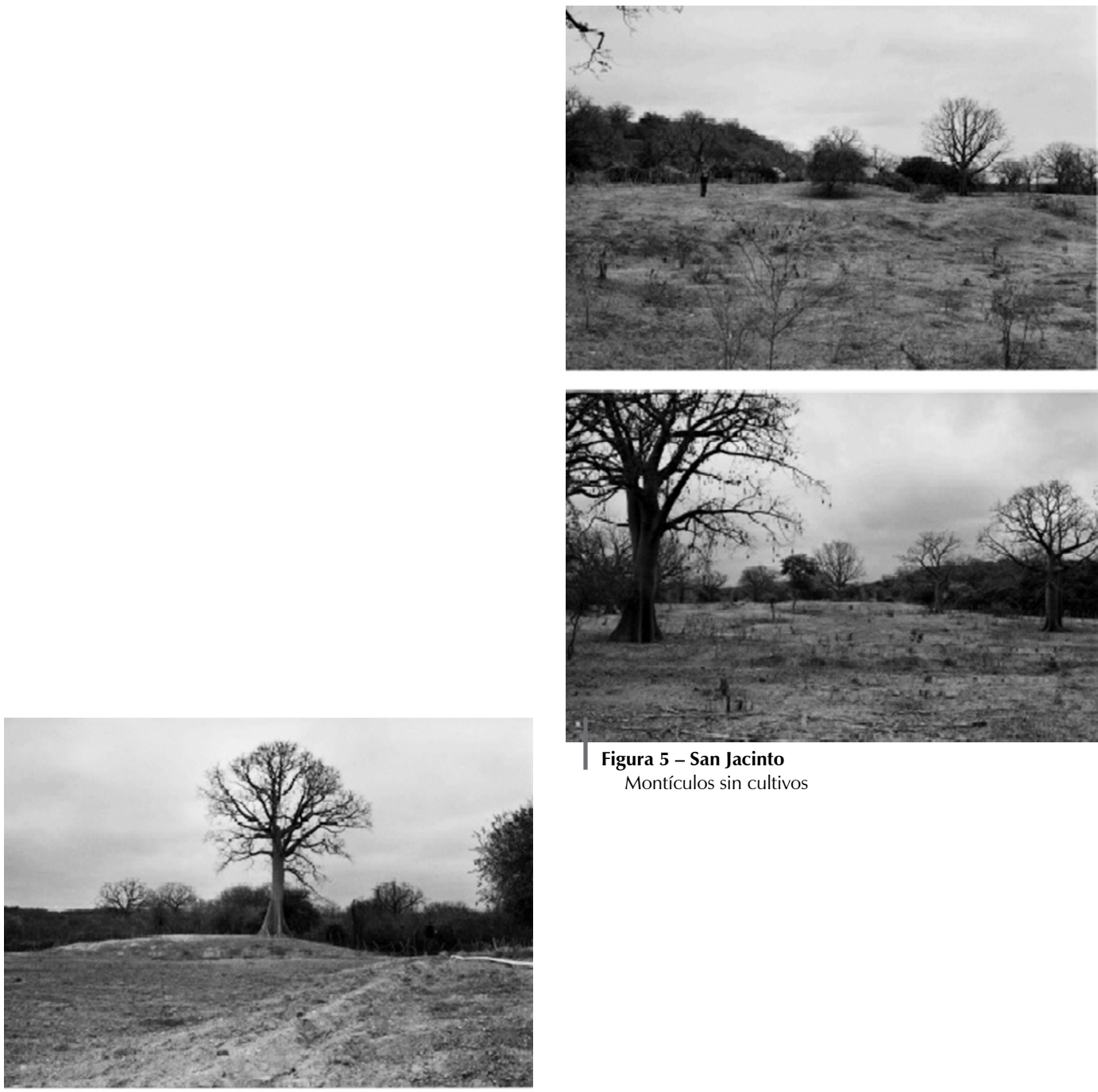

Figura 5 - San Jacinto

Montículos sin cultivos

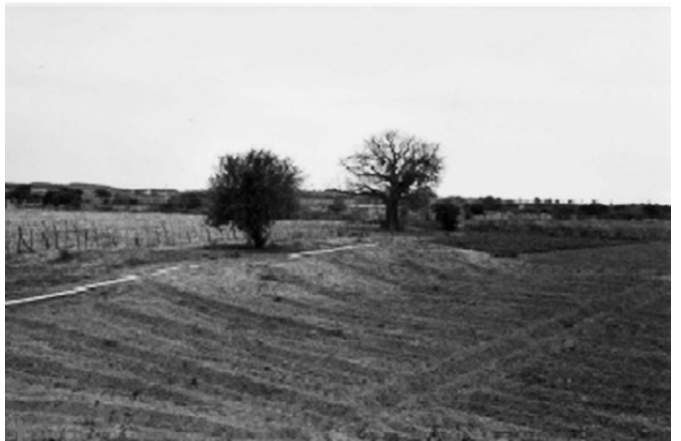

Figura 6 - San Jacinto

Vista de los montículos arados (2003) 
de agua dulce (río, esteros, una posible paleo-laguna y todo el sistema del estuario de Porto Viejo). Considerando que la distancia entre las ciudades de Manta y Bahía de Caráquez (ambas ocupadas en la época prehispánica) alcanzaba $90 \mathrm{~km}$, no sería nada extraño que entre las dos, haya existido un importante pueblo precisamente en el lugar más propicio de esta costa.

Parece además probable que esta localización tan favorable fue también percibida aún antes de la época manteña, durante el periodo de Desarrollos Regionales, ocasionando un primer asentamiento de grupos anteriores en este mismo lugar de Japoto. Sin embargo, este punto requiere mayores estudios en un próximo futuro.

Mientras los asentamientos españoles de la época de la Conquista tenían que buscar una protección (especialmente contra los ataques de corsarios europeos) y se construyeron un poco tierra adentro alejándose de la orilla del mar10, los establecimientos indígenas manteños podían perfectamente existir sin peligro de saqueos puesto que existía una especie de «paz manteña» garantizando la tranquilidad de las poblaciones indígenas locales. Es probable que en esta región, era suficiente establecer el sitio de tal forma que era protegido de las amenazas naturales como las tormentas y de los eventuales tsunamis.

\section{1. 3. Vestigios de cerámica11}

La cerámica se compone de recipientes utilitarios, culinarios y de uso más excepcional. También, existen varios ejemplos de adornos de recipientes y de figurinas antropomorfas y zoomorfas. A raíz de las observaciones se hizo patente que una buena parte de la cerámica se asemeja a la cerámica temprana manteña de otros sitios del área manteña. Otra parte reflejaría una época más reciente. Una tercera parte refleja algunas influencias de tradiciones coetáneas —Milagro-Quevedo—o anteriores — La Tolita-Tumaco, Bahía de Caráquez-.

\section{1. 4. Vestigios óseos y malacológicos}

- Huesos humanos: varias sepulturas primarias o secundarias se han descubierto en las temporadas 2004, 2005 y 2006 (J5, J6, J7). Existen varias modalidades: esqueleto completo en decúbito dorsal, esqueleto incompleto (sin cabeza ni pies) en decúbito dorsal, paquetes funerarios agrupando los huesos en niveles superpuestos (cf. la ponencia de T. Delabarde).

- Fauna: se han descubierto restos de fauna en varias tolas. La fauna terrestre comprende mamíferos, aves y posibles reptiles. La fauna acuática comprende muchos huesos de pescado (vértebras) y escamas. La identificación ictiológica se realizó en mayo 2006, enfatizando el consumo de peces más bien de gran tamaño y propios de un medio ambiente cercano (estuario y mar litoral), pero sin excluir completamente la pesca en el mar abierto.

- Malacología (caracoles y conchas): aparentemente hubo un consumo alimenticio de varios moluscos. Se han descubierto grandes cantidades de caracoles terrestres («caracol de monte») y conchas de ambiente acuático salobre (anadara o sea la conocida «concha negra» típica de los pisos lodosos en los manglares) o marítimo (bivalvos comestibles, madre perla, espóndilo, etc.). Pero también hemos de señalar el uso muy frecuente de conchas como materia prima para fabricar adornos corporales (chaquiras, cuentas, colgantes, etc.) y objetos (por ejemplo, un cuenco hecho en concha recortada de «malea gigas» en la tola 4 B). Sobre esta tema es de muy especial importancia el hallazgo en la tola J4 A de más de 3 mil cuentas hechas en concha, perforadas (ver la ponencia de M. Guinea presentada en el simposio ARQ 27, «moluscos arqueológicos»).

\footnotetext{
${ }^{10}$ Como fue el caso de la ciudad de Puerto Viejo, por ejemplo.

11 Ver la ponencia de K. Stothert.
} 
No entrararemos en detalles sobre todos los aspectos especiales que corresponden a las ponencias de estos varios investigadores del proyecto que presentan en este simposio sus resultados ${ }^{12}$. Solamente queremos detallar aqui un aspecto que se refiere a la construcción de las tolas que conforman el sitio.

\section{2. El proceso de construcción de las tolas}

Esta hipótesis se propone a partir de las observaciones en las tolas J3, J4, J5 y J613. En todas, se nota de forma recurrente una sucesión de capas horizontales bien compactadas y conformadas de relleno alternando con «suelos» (generalmente más delgados) que serían los niveles de ocupación humana. En la tola J3 se nota un gran número de capas con esta sucesión y ocurre lo mismo en las tolas J4 A, J5 y J6. Usualmente, en los pisos ocupados se descubren varios «rasgos» (fogones, postes, áreas de cenizas, tierra quemada, «hornos Manabítas», etc.). Pero es notable la ausencia o la escasez de cerámica en estos pisos, mientras son más frecuentes en las capas «de relleno». Pensamos que la cerámica quebrada y otros desperdicios se evacuaron en «basureros» periféricos a la zona de vivienda (tal como se nota en el caso de la tola J5 donde apareció un área periférica con un extenso basurero).

Pensamos que los constructores de las tolas acumularon cierta cantidad de relleno compactado para obtener una plataforma elevada. Sobre esta plataforma horizontal se desarrollaban sus actividades «domésticas» dentro o fuera de las viviendas. Es recurrente observar que estos rellenos son formados por capas horizontales superpuestas que forman una estratigrafía compleja. Después de cierto tiempo, probablemente al ocurrir eventos climáticos desastrosos, como eventos el Niño, la plataforma ocupada tenía que ser renovada y consolidada mediante el aporte de nuevos rellenos compactados. Al repetirse estas remodelaciones sucesivas se elevaron los montículos de forma tal que las tolas llegaron a tener varios metros de altura. En cambio, a juzgar por el gran espesor del último relleno (o sea los niveles más altos) parece que los habitantes de la fase tardía, al edificar la última plataforma, amontonaron una gran cantidad de una sola vez por motivos aún desconocidos (¿Necesidad práctica? ¿Papel simbólico?).

Esta hipótesis muestra que los manteños tenían un excelente conocimiento de la resistencia de sus construcciones de tierra apisonada en condiciones normales secas y en condiciones excepcionales muy lluviosas. Superponer una alternación de pisos compactados hechos con componentes distintos podía limitar la erosión de los montículos y las infiltraciones durante las épocas de lluvias.

Mencionaremos también un tipo de piso muy especial, descubierto en toda la cumbre de la tola J5. Desde luego parece que corresponde a la última ocupación prehispánica. En toda la superficie de la plataforma de la cumbre, la capa horizontal superior, debajo de los 10 a 15 centímetros de tierra húmica superficial, consiste en un piso hecho de tierra arcillosa quemada intencionalmente. Desde luego, no se trata de huellas de un incendio sino de un deseo de construir un piso más duradero que una sencilla capa de tierra apisonada. Es probable que esto sea también un intento de proteger este montículo por motivos que quedan por determinar. Además, es de notar que existe en la periferia un pequeño reborde vertical que pudo unir

12 P. Usselmann (geodinámica de la costa de Manabí Central)

M. Guinea (artefactos especiales: ponencias Arq. 14 y Arq. 27)

T. Delabarde (Antropología funeraria en Japoto, tola J7)

A. Touchard (Ocupaciones de la Tola J6)

K. Stothert (Cerámica de Japoto: interpretaciones)

C. Caillavet (Datos etnohistóricos sobre la costa de Manabí central)

13 Estas tolas fueron excavadas bajo la responsabilidad de los siguientes investigadores. Tola J3: M. Guinea, M. A Barriuso; tola J4 a y J4 b: M. A. Barriuso, A. Alonso; tola J5: T. Lopez y A. Touchard; Tola J6: A. Touchard; tola 7: T. Delabarde (temporadas 2004, 2005, 2006). 
este piso a las paredes verticales de la estructura doméstica que ocupó la plataforma. Lo que sugiere que puede corresponder a una práctica especial destinada a evitar infiltraciones entre las paredes y el piso interior y/o la penetración de animales y plagas desde el exterior.

\section{CONCLUSIÓN}

Varias de las ponencias específicas que se presentarán a continuación sobre el sitio de Japoto, permitirán detallar aspectos de las actividades cotidianas, artesanales o ceremoniales que nuestro proyecto permite descubrir a través de los vestigios que se han puesto a luz. Otras nos explican algunas de las relaciones del hombre prehispánico con su entorno y la forma de aprovecharlo. Es de esperar que al terminarse este estudio, podremos ofrecer aún más detalles sobre los sitios descubiertos por los conquistadores en esta costa.

\section{Referencias citadas}

CUMMINS, T., BURGOS CABRERA, J. \& MORA HOYOS, C., 1996 - Huellas del Pasado. Los sellos Jama Coaque, 254 p.; Guayaquil: Banco Central del Ecuador. Miscelánea Antropológica. Serie Monográfica 11.

ESTRADA, E., 1962 - Arqueologia de Manabí central, 210 p.; Guayaquil: Ed. Museo V. E. Estrada.

SAVILLE, M., 1907 - The antiquities of Manabí, vol. I; New York: Heye Museum.

SAVILLE, M., 1910 - The antiquities of Manabí, vol. II. I; New York: Heye Museum.

ZEIDLER, J. A. \& PEARSALL, D. M., 1994 - Regional archaeology in northern Manabí, Ecuador, vol. 1, 224 p.; Pittsburgh/Quito: University of Pittsburgh. Memoirs in Latin American Archaeology 8. 